\title{
Scalloping Correction in TOPS Imaging Mode SAR Data
}

\author{
Steffen Wollstadt, Pau Prats, Member, IEEE, Markus Bachmann, Josef Mittermayer, and Rolf Scheiber
}

\begin{abstract}
This letter presents an investigation on scalloping correction in the Terrain Observation by Progressive Scan (TOPS) imaging mode for synthetic aperture radar systems with electronically steered phased array antennas. A theoretical simulation of the scalloping is performed, and two correction methods are introduced. The simulation is based on a general cardinal sine (sinc) antenna model as well as on the TerraSAR-X (TSX) antenna model. Real TSX data acquired over rainforest are used for demonstration and verification of the scalloping simulation and correction. Furthermore, a calibration approach, taking into account the special TOPS imaging mode properties, is introduced.
\end{abstract}

Index Terms-Electronic beam steering, phased array antenna, scalloping, Terrain Observation by Progressive Scan (TOPS), TerraSAR-X (TSX), TOPS calibration.

\section{INTRODUCTION}

$\mathbf{T}$ HE HIGH flexibility of the TerraSAR-X (TSX) instrument commanding allows for experimental synthetic aperture radar (SAR) acquisitions [1]. Therefore, it is possible to execute special instrument commands required for data acquisition in the Terrain Observation by Progressive Scan (TOPS) imaging mode [2]. This mode was first demonstrated in space with TSX [3] and is selected to be the default mode in the upcoming Sentinel-1 mission [4], [5]. The TOPS mode provides wide swath imaging with greatly improved performance w.r.t. scalloping, signal-to-noise ratio, and azimuth ambiguity ratio compared to the ScanSAR imaging mode [6].

The TOPS acquisition mode is a burst imaging mode, which illuminates more than one subswath to obtain a large ground coverage in elevation. Each subswath is composed of several bursts [2]. In order to perform the azimuth beam steering required during each burst, either a mechanical or an electronic beam steering has to be conducted. In the case of TSX with its phased array antenna, an electronic beam steering is applied, which is advantageous w.r.t. the beam steering velocity. The drawback of electronic steering is a small scalloping effect,

Manuscript received July 13, 2011; revised October 4, 2011; accepted October 24, 2011. Date of publication December 23, 2011; date of current version May 7, 2012. This work was supported by the European Space Research and Technology Centre, European Space Agency, under Contract 22243/09/NL/JA.

The authors are with the Microwave and Radar Institute, German Aerospace Center (DLR), 82234 Oberpfaffenhofen, Germany (e-mail: steffen.wollstadt@dlr.de; pau.prats@dlr.de; markus.bachmann@dlr.de; josef.mittermayer@dlr.de; rolf.scheiber@dlr.de).

Color versions of one or more of the figures in this paper are available online at http://ieeexplore.ieee.org.

Digital Object Identifier 10.1109/LGRS.2011.2176716

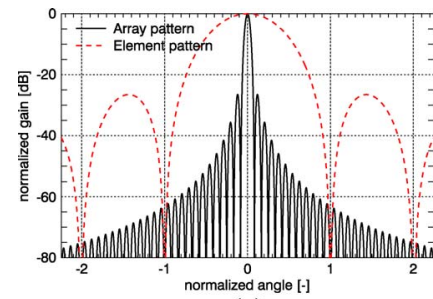

(a)

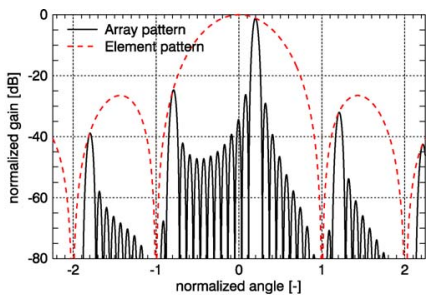

(b)
Fig. 1. (Solid line) Array antenna pattern and (dashed line) element pattern in electronic phased array azimuth beam steering. (a) Boresight case. (b) Steering angle equal to $0.2 \lambda / d$ ( $\lambda$ : wavelength; $d$ : azimuth antenna element spacing).

which is caused by the characteristics of the phased array antenna [3]. This letter presents an analysis of this scalloping effect and two correction methods, including a verification with real TOPS SAR data. Furthermore, an additional TSX calibration step is presented, as a small systematic bias has been observed in the TSX TOPS data after the scalloping correction.

This letter is structured as follows. In Section II, the simulation approach of the scalloping is given, including the impact of steering angle quantization as well as the characteristics of the TSX beam steering. In Section III, two compensation methods for the scalloping are introduced. Finally, Section IV shows the performance of these methods by means of real TSX data. Section V describes a method to calibrate the TOPS data w.r.t. the TSX antenna model.

\section{Theory AND Simulation of ScAlloping}

Scalloping in the TOPS imaging mode is a varying image intensity due to the electronic beam steering realized by means of a phased array antenna. The pattern of an array antenna can be expressed by the array element pattern multiplied by an array factor [7]. Fig. 1 shows the impact of the element pattern on the array gain, where the maximum gain is obtained at boresight becoming reduced at steered directions [3]. This causes the scalloping effect in the TOPS SAR image. It is, in fact, different to ScanSAR scalloping, where the intensity variation is caused by the varying slices of the antenna main lobe, which illuminate targets at different azimuth positions within the burst image [6].

\section{A. Methodology}

The analysis of the scalloping in the TOPS mode requires only the consideration of a single burst as, in principle, the characteristics are repeated for every burst within each subswath. Note that small variations from subswath to subswath can occur due to different pulse repetition frequencies (PRFs) or slightly different steering angles. The simulated burst intensity 


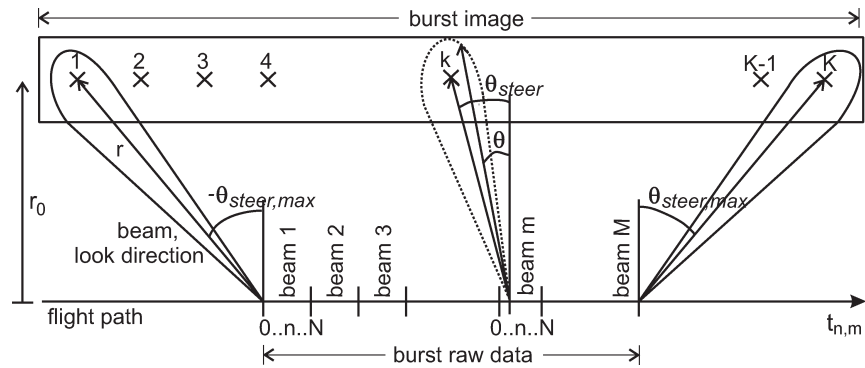

Fig. 2. TOPS acquisition geometry for the simulation of scalloping of a single burst. (Backward-looking directions mean negative angles $\theta_{\text {steer }}$.)

of a burst in the TOPS mode is based on the geometry shown in Fig. 2. The evaluation of the amplitude $a_{k}$ of a single illuminated target $k$ is described by (1). Basically, an integration of the raw data, i.e., of the received signals, is performed, where only the magnitude is considered. This means that the azimuth phase modulation is assumed to be corrected. The magnitude $g_{\text {az }}$ of the azimuth antenna amplitude pattern (one way) for each look direction is obtained by considering the varying steering direction of the different beam patterns during a single burst. This can be written as

$$
\begin{array}{r}
a_{k}=\sum_{m}^{M} \sum_{n}^{N} s\left(r_{0}, k\right)^{(n, m)} \cdot g_{\mathrm{az}}\left(\theta\left(r_{0}, k\right)^{(n, m)}, \theta_{\mathrm{steer}, \mathrm{TX}}^{(m)}\right) \\
\cdot g_{\mathrm{az}}\left(\theta\left(r_{0}, k\right)^{(n, m)}, \theta_{\mathrm{steer}, \mathrm{RX}}^{(m)}\right)
\end{array}
$$

where $s\left(r_{0}, k\right)$ represents the received echo signal except for $g_{\mathrm{az}}$ and is set to one for the sake of simplicity. $r_{0}$ is the slant range in the zero-Doppler plane, $N$ is the number of pulses per beam, and $M$ is the number of beams per burst. Therefore, $n$ and $m$ represent the azimuth time. The steering angle of the beam $\theta_{\text {steer }}^{(m)}$ defines the azimuth steering direction of the pattern for the transmit (TX) and receive (RX) cases. The look direction or azimuth antenna pattern angle $\theta\left(r_{0}, k\right)$, under which the target sample $k$ is seen, is limited by the processed beamwidth $\theta_{\text {proc }}$

$$
\theta_{\text {steer }}^{(m)}-\frac{\theta_{\text {proc }}}{2} \leq \theta\left(r_{0}, k\right)^{(n, m)} \leq \theta_{\text {steer }}^{(m)}+\frac{\theta_{\text {proc }}}{2} .
$$

According to (1) and Fig. 2, the quantized steering of the antenna main lobe follows a sequence of beams with different steering angles for each target. This means that, first, the amplitudes of all pulses in the first beam are summed up for each illuminated target sample. Second, this is repeated for every beam. This sum over all beams results in an amplitude for each target sample. A calculation for all targets $k$ is necessary to obtain the burst intensity characteristic (see Fig. 3).

\section{B. Quantization of Azimuth Beam Steering Angles}

The azimuth steering angle quantization is dependent on the instrument capabilities. In TSX, a constant steering angle is kept for a number of pulse repetition intervals (PRIs), i.e., a quantization of the steering angle is performed. Generally, the optimal case is a beam steering angle adaptation for each PRI. Therefore, the smallest possible quantization is given by the PRF. In this case, the steering is assumed to be continuous.

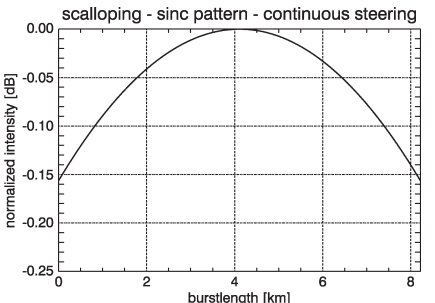

(a)

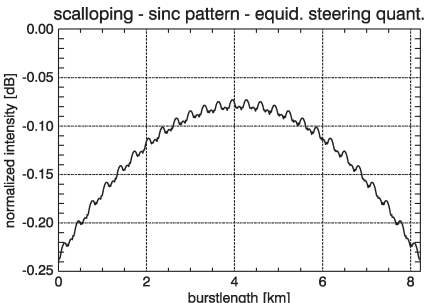

(b)
Fig. 3. Burst image intensity for (a) continuous azimuth beam steering and (b) equidistantly quantized azimuth beam steering. Beam patterns based on sinc antenna model. (a) and (b) are normalized w.r.t. (a).

TABLE I

PARAMETERS OF TOPS Data TAKE OVER AMAZONIAN RAINFOREST

\begin{tabular}{ll}
\hline Parameter & Value, [Subswath 1/2/3/4] \\
\hline Center coordinate (lat./lon.) & $-7.8785^{\circ} /-68.6805^{\circ}$ \\
Total scene size (range x azimuth) & $100 \mathrm{~km} \mathrm{x} 65 \mathrm{~km}$ \\
Pulse repetition frequency & $\approx 4000 \mathrm{~Hz}$ \\
Incidence angle / mid slant range & $33^{\circ} / 600 \mathrm{~km}$ \\
Azimuth resolution & $16 \mathrm{~m}$ \\
Number of bursts & {$[8 / 8 / 8 / 8]$} \\
Number of azimuth beams (in each burst) & {$[38 / 23 / 25 / 36]$} \\
Number of pulses per azimuth beam & {$[27 / 49 / 42 / 29]$} \\
Maximum azimuth steering angle & {$[0.48 / 0.43 / 0.47 / 0.46]^{\circ}$} \\
Nominal steering angle quantization & {$[0.026 / 0.039 / 0.039 / 0.026]^{\circ}$} \\
\hline
\end{tabular}

In order to simulate the different azimuth beams, an array antenna model is used, where the single element pattern is based on a sinc function $(\sin (x) / x)$. The magnitude can be described by [7]

$$
\begin{aligned}
& g_{\mathrm{az}}=\sqrt{G_{0}} \cdot \operatorname{sinc}\left(\frac{d}{\lambda} \pi \sin (\theta)\right) \\
& \cdot \sqrt{\frac{W_{\mathrm{el}}}{W_{\mathrm{az}}}} \cdot \mid \sum_{w=1}^{W_{\mathrm{az}}} e^{j \frac{2 \pi}{\lambda} w d\left(\sin (\theta)-\sin \left(\theta_{\mathrm{steer}}\right)\right) \mid}
\end{aligned}
$$

where $\lambda$ is the wavelength, $d$ is the azimuth spacing between the phase centers of the antenna elements, $G_{0}$ is the antenna element power gain, and $W_{\mathrm{az}}$ and $W_{\mathrm{el}}$ are the numbers of azimuth and elevation antenna elements, respectively. For TSX, $W_{\mathrm{az}}$ is $12, W_{\mathrm{el}}$ is 32 , and $d$ is $0.4 \mathrm{~m}$. The sinc function represents the element factor, and the sum over the exponential function represents the array factor. The resulting two-way gain is shown in Fig. 1.

The simulation of a continuous beam steering with the applied data take configuration in Table I, i.e., a TSX azimuth steering range of $\left[-0.48^{\circ}, 0.48^{\circ}\right]$, typical in the case of four subswaths, results in the burst intensity characteristic, i.e., the scalloping [Fig. 3(a)]. It follows directly from the variation of the antenna element factor, shown in Fig. 1. The absolute difference, i.e., the scalloping, is around $0.15 \mathrm{~dB}$. An azimuth beam steering with 38 azimuth beams per burst and an equidistant quantization of $0.026^{\circ}$ results only in a small periodic deviation of the intensity characteristic and a slightly lower absolute intensity, as can be seen in Fig. 3(b). Details about the influence of different quantizations are investigated in [3]. Note that the scalloping would be around $0.5 \mathrm{~dB}$ for the maximum nominal steering angle of TSX $\left(0.75^{\circ}\right)$. 


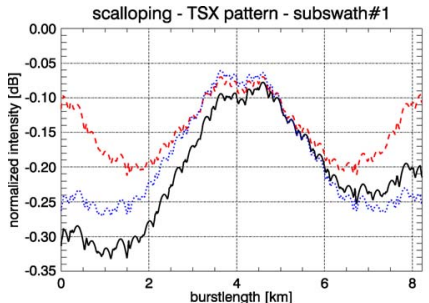

(a)

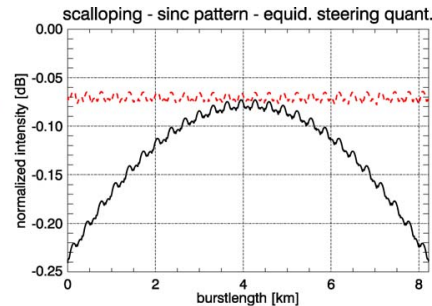

(b)
Fig. 4. Burst image intensity. (a) For (black/solid) TSX beam steering angle quantization with TSX reference pattern, (blue/dotted) TSX beam steering angle quantization with sinc-function-based antenna model, and (red/dashed) corrected intensity. (b) For (black/solid) equidistantly quantized beam steering with sinc-function-based model and (red/dashed) corrected intensity. (a) and (b) are normalized w.r.t. Fig. 3(a).

\section{Azimuth Beam Steering of TSX}

As TSX was not designed for the TOPS imaging mode, it is a challenge to optimize the TOPS acquisition parameters in order to meet the desired performance requirements, taking into account the instrument constraints. The same real data take configuration parameters (see Table I) as those used in Section II-B are also used for the simulation in this section. The crucial difference is the application of the TSX reference antenna patterns (see Section V-A). The result in Fig. 4(a) (solid line) shows a clearly visible scalloping in the range of $0.25 \mathrm{~dB}$. Compared to Fig. 3(b), the scalloping characteristic is deviating, although the steering parameters in both simulations were equal. These deviations can be explained by a closer look at the reference patterns. The first deviation, the difference in the gain level at the beginning $(-0.3 \mathrm{~dB})$ and the end $(-0.2 \mathrm{~dB})$ of the burst, can be explained by a slight maximum gain bias between the forward- and backward-looking beams, i.e., the element pattern is not perfectly symmetrical. The reference beams looking backward have up to $0.1 \mathrm{~dB}$ less gain than the corresponding forward-looking ones. Note that these variations are on the same order of magnitude as the accuracy of the antenna pattern gain, i.e., $\sim 0.2 \mathrm{~dB}$ (see Section V-A). The second deviation is the intensity shape variation, which follows from the fact that the resulting beam steering angle quantization of the reference antenna patterns is nonequidistant. The quantization varies between $0.0185^{\circ}$ and $0.0305^{\circ}$ with a mean of $0.026^{\circ}$, which is identical with the nominal quantization of this subswath. This can be verified by using the sinc-function-based antenna model from Section II-B, together with the varying steering angle quantization, yielding the same characteristic, shown in Fig. 4(a) (dotted/blue line). It can be concluded that a coarse nonequidistant steering angle quantization has a considerable impact on the scalloping characteristic.

\section{Correction OF SCALloping}

\section{A. Correction on Raw Data Level}

This method for scalloping correction is based on an equalization of the individual pattern amplitude of each steered azimuth beam and is applied to the SAR raw data directly. For each steered azimuth beam, the ratio of the maximum amplitude

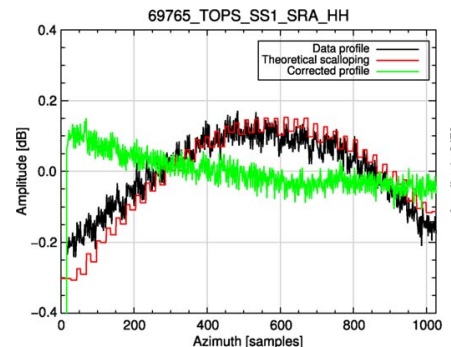

(a)

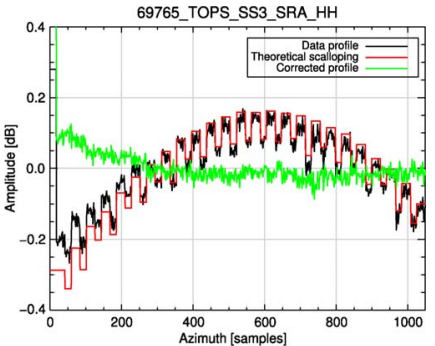

(b)
Fig. 5. Scalloping correction with scalar factor at raw data level for subswaths 1 and 3 [(a) and (b)]. (Black) Normalized raw data profile, (red) theoretical profile computed with the reference antenna patterns, and (green) profile after the correction.

of the boresight beam, chosen as reference, and the maximum amplitude of the steered beam is calculated with

$$
c_{\mathrm{TX} / \mathrm{RX}}^{(m)}=\frac{g_{\mathrm{az}}\left(\theta\left(r_{0}, k\right)^{(n, m)}=0, \theta_{\mathrm{steer}, \mathrm{TX} / \mathrm{RX}}^{(m)}=0\right)}{g_{\mathrm{az}}\left(\theta\left(r_{0}, k\right)^{(n, m)}=\theta_{\mathrm{steer}, \mathrm{TX} / \mathrm{RX}}^{(m)}, \theta_{\mathrm{steer}, \mathrm{TX} / \mathrm{RX}}^{(m)}\right)} .
$$

Finally, the antenna pattern amplitude of each steered azimuth beam is weighted over its whole beamwidth $\theta_{\text {proc }}$ by the same resulting scalar compensation factor $c_{\mathrm{TX} / \mathrm{RX}}^{(m)}$, representing always the ratio of the maximum amplitudes. According to (1), the compensated burst amplitude for one target sample is then

$$
\begin{array}{r}
a_{k}=\sum_{m}^{M} \sum_{n}^{N} s\left(r_{0}, k\right)^{(n, m)} \cdot c_{\mathrm{TX}}^{(m)} \cdot g_{\mathrm{az}}\left(\theta\left(r_{0}, k\right)^{(n, m)}, \theta_{\mathrm{steer}, \mathrm{TX}}^{(m)}\right) \\
\cdot c_{\mathrm{RX}}^{(m)} \cdot g_{\mathrm{az}}\left(\theta\left(r_{0}, k\right)^{(n, m)}, \theta_{\mathrm{steer}, \mathrm{RX}}^{(m)}\right) \cdot
\end{array}
$$

The compensation factors for all beams in a single burst are shown in Fig. 5 (red line). Obviously, it represents also the theoretical raw data intensity distribution within a burst. Fig. 4(b) shows that an almost perfect compensation is possible for the continuous and equidistantly quantized cases. The application of the correction to the TSX case results in the burst intensity shown in Fig. 4(a) (red/dashed). The main scalloping effect induced by the antenna element pattern can be compensated, but the intensity variation due to the nonequidistant beam steering still remains.

\section{B. Correction on Image Data Level}

The second method for the correction of the scalloping is the direct application of the simulated scalloping intensity (Fig. 4(a), solid line) on the focused image data, i.e., on each azimuth burst line. Assuming that the simulated scalloping for all bursts in the corresponding subswath is valid, the multiplication of the focused data with the inverse of the simulated scalloping intensity will obviously result in a complete removal of the scalloping characteristics, including the nonideal quantization effect imposed by the TSX patterns. Therefore, the level of compensation is determined by the overall simulation accuracy and the antenna pattern accuracy. In summary, this method implies an additional step, namely, the simulation of the scalloping and its application to the image data after SAR processing, 


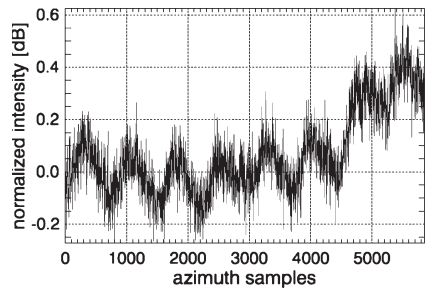

(a)

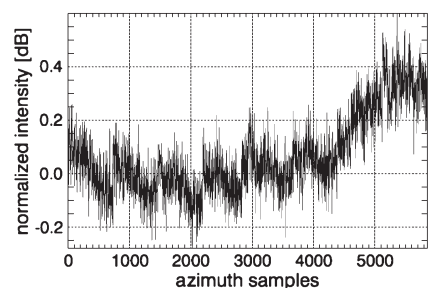

(c)

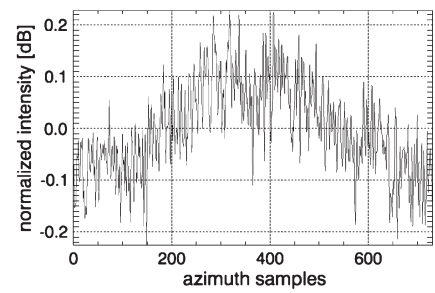

(b)

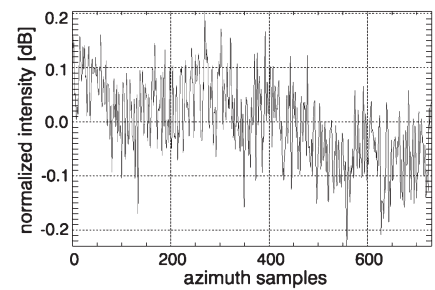

(d)
Fig. 6. Azimuth image profile of the rainforest data take. (a) Subswath 1 (eight bursts) with scalloping and (c) after correction on raw data level. (b) Burst 3 (subswath 1) with scalloping and (d) after correction on raw data level.

contrary to the former method, which is applied directly to the raw data before SAR processing.

\section{Demonstration of CorRection With TSX DatA}

A TSX TOPS data take over the Amazon Rainforest in Brazil, South America, with relatively homogeneous backscatter was acquired to verify the correction methods. Table I contains the acquisition parameters.

\section{A. Demonstration of Correction on Raw Data Level}

In Fig. 5, the computed theoretical scalloping using the TSX reference patterns, the measured azimuth raw data profile for all bursts, and the final corrected profile are shown. The staircase effect is caused by the beam steering angle quantization. Furthermore, the jumps downward within the staircase profile are caused by changing the beams between pulse transmission and reception, which always differ by the number of traveling pulses. This has to be taken into account since the TX and RX patterns are not equal during these pulses. The figure proves a very good correlation between the theoretical scalloping and the one measured in the raw data. In particular, the jump sequence fits very well with the real data profile. This is more apparent in subswath 3, where the number of switched azimuth beams is smaller (cf. Table I). A small deviation between both profiles can be observed for the negative squint angles. This becomes evident in the corrected profiles (green line). The overcorrection results from a slight deviation in the azimuth element pattern, i.e., the backward-looking reference patterns have less amplitude than the real beams. This observation could be confirmed by a second independent TOPS rainforest data take at a different date. Considering the TSX antenna model (Section V-A), this systematic variation of up to $0.15 \mathrm{~dB}$ in Fig. 5 is well within the specifications for the antenna patterns $( \pm 0.2 \mathrm{~dB})$.

The scalloping in the azimuth image profile in Fig. 6(a) is clearly visible in each of the eight bursts. Note that the last

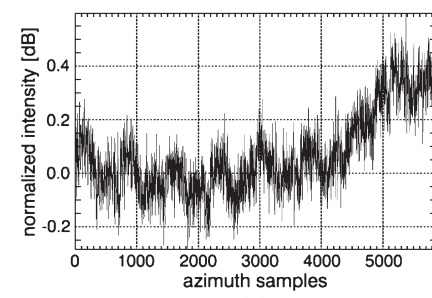

(a)

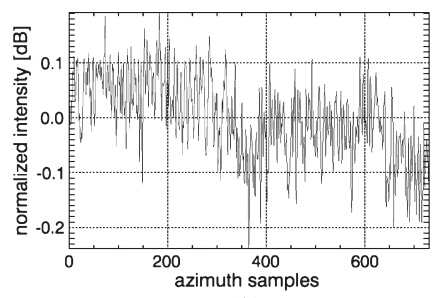

(b)
Fig. 7. Azimuth image profile after the correction on image data level (a) Subswath 1. (b) Burst 3 of subswath 1.

two bursts still show scalloping but the overall backscatter level is increased due to some heterogeneity in the scene. Fig. 6(b) shows the third burst in more detail. It shows again, compared to the simulated burst intensity shown in Fig. 4(a) (solid line), that the measured backward-looking beam intensity is slightly higher than the one of the TSX reference patterns. The nonequidistant quantization effect, i.e., the specific burst shape, is observable but not very distinctive. In the corrected azimuth image profile in Fig. 6(c) (eight bursts) and (d) (third burst), the effect of overcorrection detected in the raw data profile can be observed again at the negative steering angles in all bursts.

\section{B. Demonstration of Correction on Image Data Level}

Fig. 7(a) shows the corrected azimuth image profile based on the correction on image data level. It shows indeed a removed scalloping compared to Fig. 6(a) but still an overall intensity variation on the same order of magnitude as the former scalloping, i.e., $0.2 \mathrm{~dB}$ relative to the mean backscatter. This results from the assumption of a homogeneous scene and using always the same correction function or theoretical scalloping intensity, respectively, for all bursts. However, in fact, the shape of each burst is slightly different from the simulated scalloping intensity, and the scene is not totally homogeneous, as already stated in Section IV-A. Similar as in Section IV-A, the overcorrection can be observed again in all bursts, e.g., burst 3 [Fig. 7(b)].

\section{TOPS CALIBRATION AND TSX ANTENNA MOdEL}

The residual image intensity variation in the scallopingcorrected TOPS bursts in Section IV shows that the TOPS mode needs a very precise calibration w.r.t. the steered azimuth beams. This section shows that the observed bias can be used either to correct this residual variation or to refine the calibration of the reference antenna patterns of the steered azimuth beams. Therefore, this raw data correction turns out to be a very advantageous calibration method to calibrate many different steered azimuth beams at once.

\section{A. TSX Antenna Model}

The TSX reference patterns consist of a set of antenna patterns for all available azimuth beams of the TSX instrument with a pointing accuracy of $0.002^{\circ}$ in flight direction. These patterns are synthesized patterns and are based on an accurate 


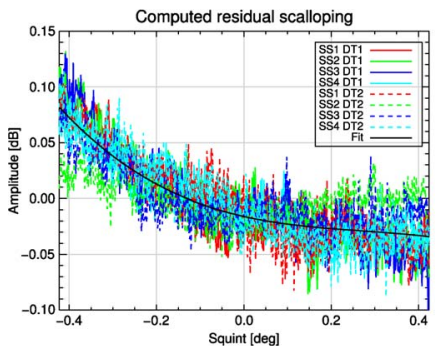

(a)

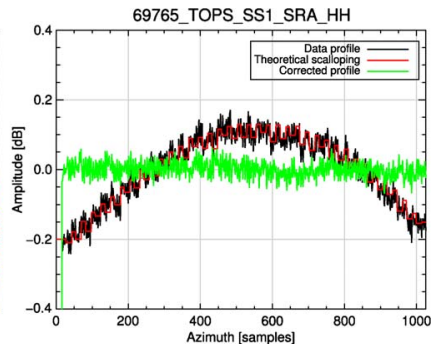

(b)
Fig. 8. (a) Calibration function averaged over scalloping-corrected raw data profiles of eight subswaths. (b) Scalloping-corrected and calibrated raw data profile of subswath 1 .

mathematical antenna model [8]. This approach was chosen due to the huge amount of more than 10000 possible beams of TSX and, at the same time, its flexibility to modify these or to even design new beams in orbit without recalibration. This is possible by employing, first, the on-ground measured antenna patterns of the individual subarrays, the so-called embedded patterns, and, second, the actual beam excitation coefficients defining the beam. The requirement of $\pm 0.2 \mathrm{~dB}$ for the accuracy of the beam gain variation was verified for the boresight azimuth pattern but not explicitly for steered azimuth beams and, therefore, not for the beam-to-beam gain variation in azimuth.

\section{B. TOPS Calibration and Application to TSX Data}

The raw data profiles presented in Fig. 5 show a very small systematic error characteristic w.r.t. the profile from the reference patterns. In order to correct this behavior, a common offset could be determined by several homogeneous rainforest acquisitions. Differences in the absolute scalloping due to different maximum steering angles have to be considered as well as incidence angle dependences. A calibration function can be retrieved by averaging, which can be applied to all TOPS data sets in order to overcome the residual bias. In the case of raw data correction, the calibration function is applied directly to the data before SAR focusing. In the case of image data correction, this offset has to be included into the scalloping simulation from which the scalloping correction function for the image data is obtained.

For demonstration purposes, a fit over the corrected profiles of eight subswaths (two scenes) was performed covering a large incidence angle range and approximately the same maximum steering angles [Fig. 8(a)]. The scalloping- and bias-corrected raw data profile of subswath 1 is shown in Fig. 8(b). Finally, Fig. 9 shows the corresponding azimuth image profile.

\section{CONCLUSION}

In principle, the TOPS mode as a wide swath imaging mode benefits from the absence of scalloping. However, in the case of electronic beam steering, a small scalloping is present, resulting from the antenna element pattern. This letter has shown two methods for overcoming this effect, one based on raw data

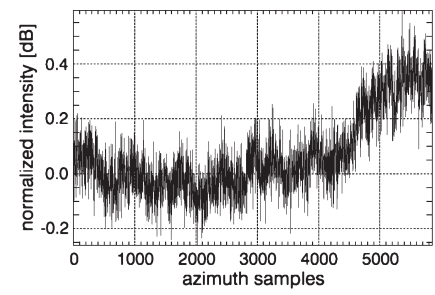

(a)

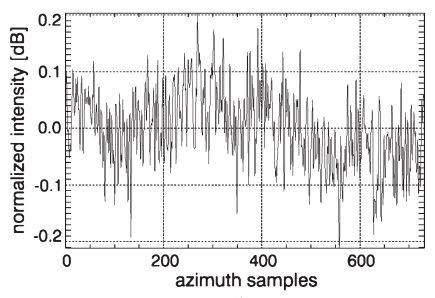

(b)
Fig. 9. Azimuth image profile after correction on raw data level and calibration. (a) Subswath 1. (b) Burst 3 of subswath 1.

level and the other based on image data level. The approaches were tested and validated with simulated as well as with real TSX data. Both the simulated scalloping and the measured scalloping are very small $(0.2 \mathrm{~dB})$. Therefore, despite the very good absolute antenna model accuracy $(\leq 0.2 \mathrm{~dB})$, the relative accuracy of the azimuth patterns is the limiting factor for the scalloping correction. However, generally, scalloping correction is desired, since larger maximum steering angles imply also a larger scalloping. Both correction methods show a very good performance with TSX data and yield comparable results. The raw-data-based method is more promising, as it can be applied directly to the data prior to SAR processing, whereas the image-data-based method requires an additional simulation step and a SAR postprocessing correction. Additionally, the raw data correction can be easily verified by an equalized raw data profile, which provides the opportunity for a databased calibration approach. In order to correct for a very small systematic bias in the gain of the backward-steered azimuth beams within the absolute TSX antenna model accuracy, several averaged scalloping-corrected raw data profiles were used to derive a correction function and to radiometrically correct the TOPS acquisition. This avoids a recalibration of each single beam and can be also used for an efficient calibration of all steered azimuth beams of a SAR system.

\section{REFERENCES}

[1] J. Mittermayer, M. Younis, R. Metzig, S. Wollstadt, J. Marquez Martinez, and A. Meta, "TerraSAR-X system performance characterization and verification," IEEE Trans. Geosci. Remote Sens., vol. 48, no. 2, pp. 660-676, Feb. 2010

[2] F. De Zan and A. Monti Guarnieri, "TOPSAR: Terrain Observation by Progressive Scans," IEEE Trans. Geosci. Remote Sens., vol. 44, no. 9 , pp. 2352-2360, Sep. 2006.

[3] A. Meta, J. Mittermayer, P. Prats, R. Scheiber, and U. Steinbrecher, "TOPS imaging with TerraSAR-X: Mode design and performance analysis," IEEE Trans. Geosci. Remote Sens., vol. 48, no. 2, pp. 759-769, Feb. 2010.

[4] E. Attema, M. Davidson, P. Snoeij, B. Rommen, and M. Floury, "Sentinel-1 mission overview," in Proc. IEEE IGARSS, Cape Town, South Africa, Jul. 2009, pp. I-36-I-39.

[5] R. Scheiber, S. Wollstadt, S. Sauer, E. Malz, J. Mittermayer, P. Prats, P. Snoeij, and E. Attema, "Sentinel-1 imaging performance verification with TerraSAR-X," in Proc. EUSAR, Aachen, Germany, Jun. 2010, pp. 1-4.

[6] A. Meta, P. Prats, U. Steinbrecher, J. Mittermayer, and R. Scheiber, "TerraSAR-X TOPSAR and ScanSAR comparison," in Proc. EUSAR, Friedrichshafen, Germany, Jun. 2008, pp. 1-4.

[7] C. A. Balanis, Antenna Theory-Analysis and Design, 2nd ed. New York: Wiley, 1997.

[8] M. Bachmann, M. Schwerdt, and B. Bräutigam, "TerraSAR-X antenna calibration and monitoring based on a precise antenna model," IEEE Trans. Geosci. Remote Sens., vol. 48, no. 2, pp. 690-701, Feb. 2010. 